\section{More Than One Ever Wanted To Know About X-ray Detectors Part IX: Antigravity Holes}

\author{
Mark W. Lund, Ph.D, MOXTEK, Inc.
}

In this series we have explained why $\mathrm{x}$-ray spectrometers are useful in electron microscopes and, to some extent, how they work. In this article I will discuss some of the physics behind the detector.

First I have been asked to explain what a hole is. In a silicon crystal every silicon atom is bound to four others with covalent bonds. Every electron has a home, which consists of its home atom in the case of the inner electrons. The four outer (or valence) electrons have a home on the road, shuttling back and forth between two atoms each. In this way the five atoms share eight electrons among themselves

When a phosphorus atom is put into the lattice it can participate in bonds with all the bonds of the silicon atom it replaced and have one extra electron that cannot participate in a covalent bond. This electron is easy to separate from the phosphorus atom and, after it does, it is free to roam around the crystal, but it leaves behind a fixed positive charge on the phosphorus atom's nucleus which cannot move. This extra positive charge is no longer cancelled by having the free electron close by. Likewise, a boron atom can fit into the lattice, but it lacks an electron to link up with all its silicon neighbors. It is easy for an electron from a nearby silicon atom's valence shell to flip over into this position which then makes the boron atom look as if it is charged negative. It also leaves the neighboring silicon atom without one of its electrons, which makes this atom look like a positive charge to the normally neutral lattice.

As other electrons move in to fill the gap one at a time, the electron vacancy moves in the opposite direction to the electrons. For most applications this vacancy can be considered to be a positive charge moving

\section{COST-EFFECTIVE EM MAGNETIC SHIELDINGJ}

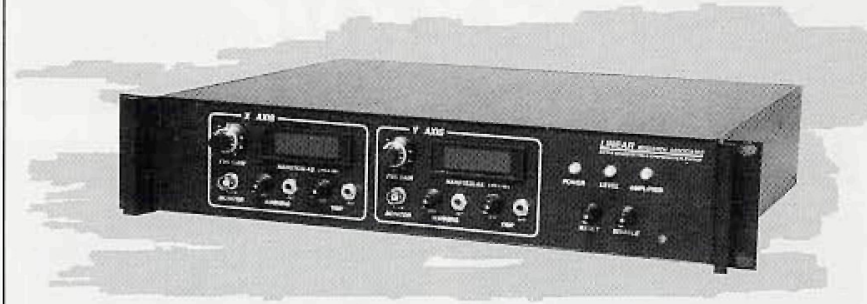

LINEAR RESEARCH ASSOCIATES' electronic active-shielding systems will dramatically improve EM performance at sites affected by fields from a.c. power wiring, ground loops, transformers and related sources. Especially recommended for FEG/PEELS installs or upgrades. Call or fax LRA for complete information describing our EMFC-series wideband a.c. magnetic field compensation sytems, or with any questions regarding LRA equipment, site surveys or EMF issues.

(607)387-3411 - FAX (607)387-7806 through the lattice. Because it is not a real particle, but the lack of a particle, it is called a hole. When a free electron from a phosphorus atom meets a hole from a boron atom, it can fill it. When it does this the electron and hole "disappear" into the lattice, because the electron takes up permanent residence in the last covalent bond that the hole appeared in and the lattice looks normal. The only evidence left is that their original boron and phosphorus "home" atoms are still charged. When boron and phosphorus dopants are uniformly scattered through the lattice the charges just cancel each other out. When they are in different parts of the crystal an electric field forms between them, which is what makes the built-in voltage of a $p-n$ junction.

Now let's consider a pure silicon crystal with an electric field on it. When an x-ray is absorbed it knocks an electron out of its covalent bond, leaving a hole behind. Left on its own it would find its home again, or one just like it. When an electric field is applied to the crystal, however, it can't find its home and moves along with the field toward the positive electrode. Likewise, the hole gets filled with another covalent electron from one of the neighboring atoms trying to move with the field, and it appears as if the hole were moving toward the negative electrode. Semiconductor workers soon tire of remembering that it is the electrons that are moving and talk about the holes as if they were the actual charge carriers with mass, mobility, lifetimes, etc. This is OK because they act just like positive charges in the crystal. Just about the only thing you can't do with holes is shoot them out into space.

Notice the fundamental difference between the way that dopants form charge carriers and the way that $x$-rays form charge carriers. When an electron or hole leaves a dopant atom, it leaves a fixed charge behind because the nucleus of the atom can't move. When an $x$-ray generated electron-hole pair leave an atom, they leave a neutral atom behind and can both move in the electric field of the detector.

An interesting thing about holes is they have negative mass--they are antigravity. Do you remember driving with a helium balloon in the car? When you slam on the brakes the balloon flies to the back of the car, just the opposite direction of the children and soft drinks. (Don't get into a wreck tying this at home; get one of the kids to drive while you experiment in the back seat.). The apparent negative mass of the balloon is due to the fact that it is less dense than the air. When the air is rushing to the front of the car, it pushes the balloon back. In the same way, since the hole is an absence of an electron it should float to the top of the crystal. A simple way to build an antigravity machine is to shoot the holes out of the crystal onto the bottom of your chair! Of course the holes don't actually float to the top of the crystal because gravity is very weak compared to the other forces in the crystal and, as I mention before, you can't remove holes from the crystal.

When an x-ray hits the detector a charge is created proportional to the $x$-ray energy. Initially this does not affect the fields inside the detector because the cloud of positive and negative local charges overlap exactly, giving a net charge of zero. Soon, however, the electrons and holes start to separate due to the applied electric field. As the charges move they induce a current to flow on the detector electrodes. This current is the signal, which is then amplified and processed to form a point in the spectrum.

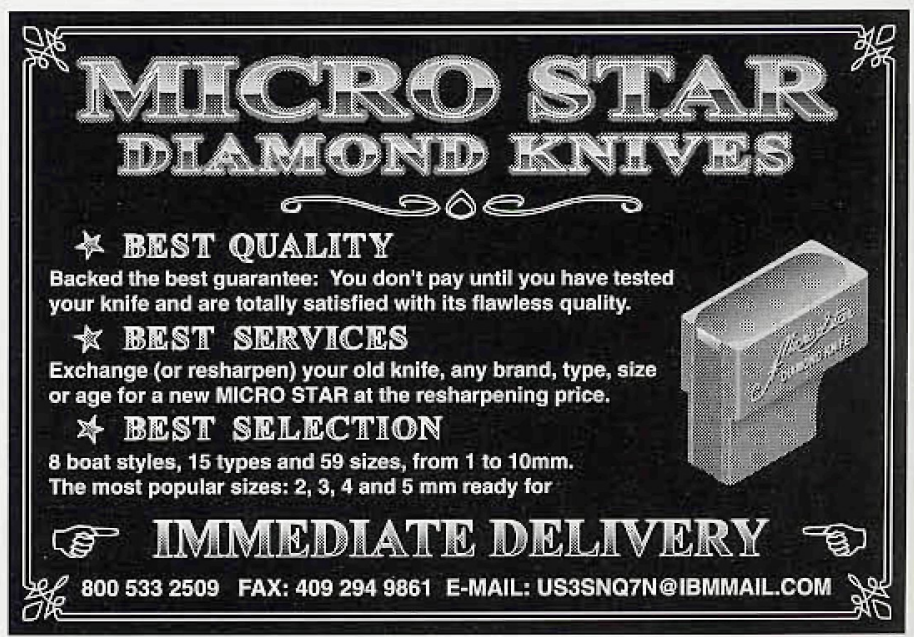




\section{LE0 435VP - first in the field}

The first fruit of the new co-operation between Leica and Zeiss is a high performance, variable pressure scanning electron microscope, the LEO 435VP.

Opening up a new world of applications including high $\mathrm{kV}$ EDX analysis of uncoated, non-conducting materials and morphological studies of porous and hydrated specimens, the LEO 435VP offers a practical and versatile tool for all SEM users.

The LE0 435VP also provides:

7. Stunning imaging performance

$\checkmark$ Total computer control

7 Precision engineered 5 -axis motorised stage

7 User-friendly interface to familiar software packages

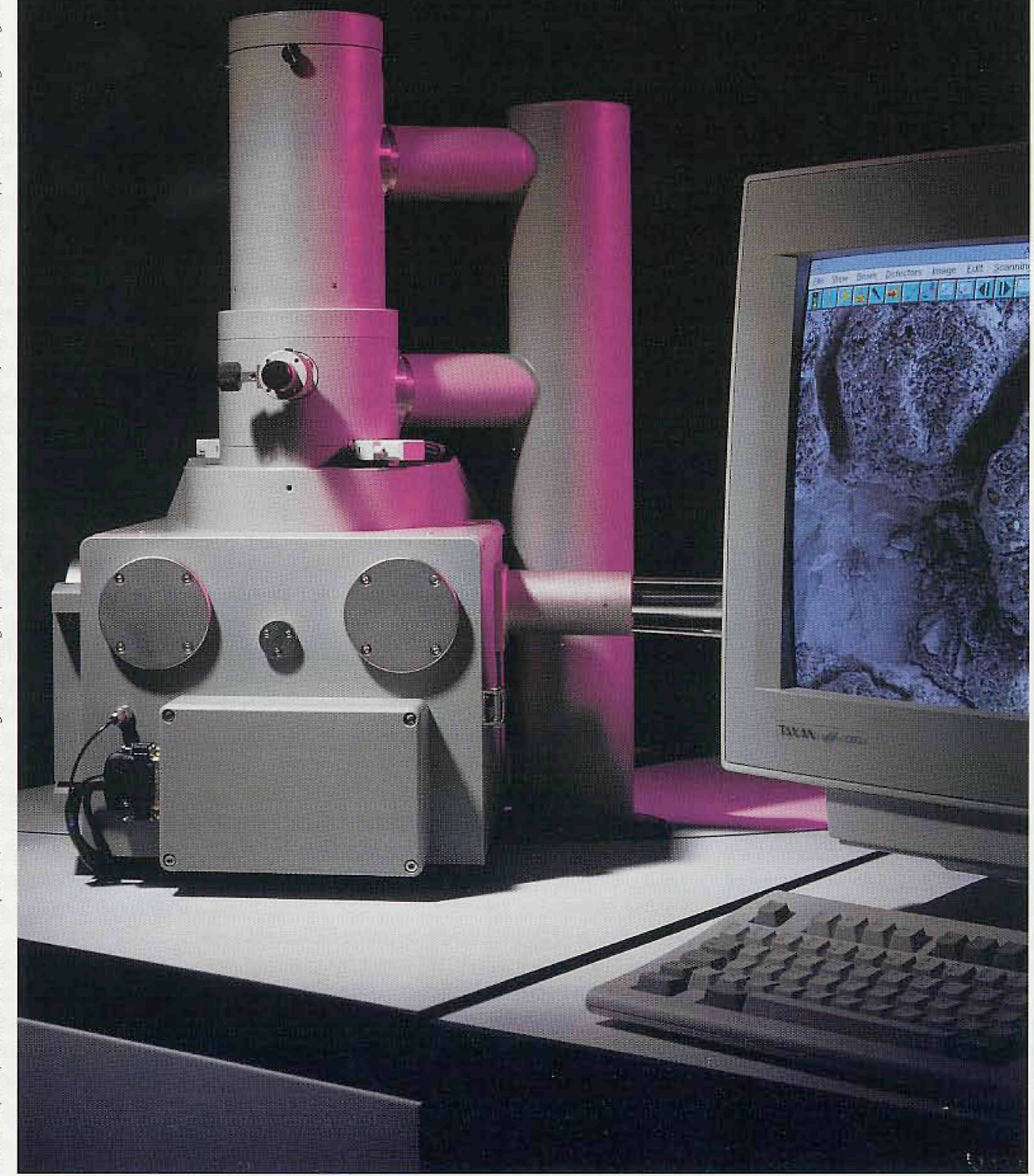

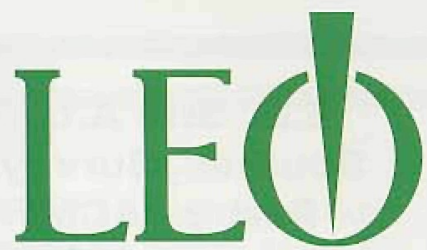

\section{A new}

\section{force in}

electron

\section{microscopy}

October 11995 marked the birth of LEO, a new

international company

formed from a co-operation between Leica and Zeiss.

Bringing together the traditional qualities and expertise of the two parent companies, LEO marks the beginning of a new era in the development, manufacture, sales and service of scanning and transmission electron microscopes.

LEO will provide worldwide coverage, serving the needs of science, industry and education.

LEO - a new force in electron microscopy and the birth of a new standard of quality, expertise and innovation.

LEO Electron Microscopy Ltd Clifton Road Cambridge $\mathrm{CB} 130 \mathrm{H}$ England

Telephone (44) 1223414166 Fax (44) 1223412776

LEO Elektronenmikroskopie $\mathrm{GmbH}$ D-73446 Oberkochen Germany Telephone (49) 7364202700

Fax (49) 7364204670

LEO Microscopie Electronicue SARL 86 avenue du 18 juin 1940 92500 Rueil-Malmaison France Telephone (33) 147328510 Fax (33) 147328590

LEO Electron Microscopy Inc One Zeiss Drive Thornwood

New York 10594 USA

Telephone (1) 9147477700 Fax (1) 9147477788 